\title{
La inserción laboral y la inclusión educativa de jóvenes con discapacidad auditiva en Chincha Alta
}

\author{
Lulayman yaykuchikaa maltakunap yaćhaynin \\ yaykuchi mana uyaliniyuwan Hanay Chinchaćhu
}

\section{Ora antagantsipage ipagetanënaniro iboingapage kara ogomentotsipongokë ira iramasitagapage magemitaripage kara timagantsigite Chincha Alta}

Recibido: 28 enero 2020 Corregido: 28 marzo 2020 Aprobado: 15 junio 2020

Shirley Mirella Vásquez León

Nacionalidad: Peruana / Universidad Femenina del Sagrado Corazón (UNIFÉ) Correo: shirleyvasquezl@unife.edu.pe. / ORCID: https://orcid.org/oooo-0003-1241-8191

Edward Faustino Loayza Maturrano

Nacionalidad: Peruana / Universidad Nacional Agraria La Molina (UNALM) Correo:edwloma@lamolina.edu.pe /ORCID: https://orcid.org/oooo-0oo2-1359-8414

\section{Resumen}

La inserción laboral y la inclusión educativa es un problema que afecta a jóvenes con discapacidad ya que se entremezcla dos situaciones sociales: la exclusión y la discriminación. El presente trabajo tuvo como objetivo calcular el grado de relación existente entre la inclusión educativa y la inserción laboral en una población post adolescente afectada por la falta de audición en Chincha Alta. Se diseñó un estudio descriptivo-correlacional con una muestra de 106 jóvenes que poseen discapacidad auditiva del distrito de Chincha Alta en Ica a quienes se les aplicaron dos instrumentos para evaluar la inclusión educativa y la inserción laboral. Los resultados muestran que existe relación moderada alta, siendo significativa, entre las variables inclusión educativa e inserción laboral de personas que están afectadas en su potencial auditivo. Se concluye que la inclusión educativa se relaciona significativamente con el aspecto laboral de personas con la audición disminuida en Chincha Alta durante el 2019.
Palabras clave:

Inserción laboral, inclusión educativa, discapacidad auditiva, habilitación laboral.

\section{Lisichiku limaykuna:} Lulayman yaykuchi, yaćhayman hukllachi, mana uyali atipaniyu, lulay yaćhay.

\section{Nibarintsipage katingatsaro:} ipagetanënani antagantsipage, kara ogomentotsipongopagekë, ira magemitaripage irantanetaigeta. 


\section{Labor Insertion and Educational Inclusion of Young People with Hearing Impairment in Chincha Alta}

\begin{abstract}
Labor insertion and educational inclusion is a problem that affects young people with disabilities as it intermingles two social situations: exclusion and discrimination. This paper aimed to calculate the degree of relationship between educational inclusion and job placement in a post-adolescent population affected by hearing loss in Chincha Alta. A descriptive-correlational study was designed with a sample of 106 young people who have a hearing impairment in the Chincha Alta district in Ica, to whom two instruments were applied to assess educational inclusion and job placement. The results show that there is a moderately high relationship, being significant, between the variables educational inclusion and labor insertion of people who are affected in their hearing potential. It is concluded that educational inclusion is significantly related to the employment aspect of people with decreased hearing in Chincha Alta during 2019.
\end{abstract}

\author{
Keywords \\ labor insertion, \\ educational inclusion, \\ hearing impairment, work \\ qualification.
}

\section{A Inserção laboral e a inclusão educativa de jovens com deficiência auditiva em Chincha Alta}

\section{Resumo}

A inserção laboral e a inclusão educativa, é um problema que afeta os jovens com deficiência, pois se mistura a duas situações sociais: exclusão e discriminação. Este trabalho teve como objetivo calcular o grau de relação que existe entre inclusão educativa e a inserção laboral em uma população pós-adolescente afetada pela perda auditiva em Chincha Alta. Um estudo descritivo-correlacional foi desenhado com uma amostra de 106 jovens com deficiência auditiva no distrito de Chincha Alta, em Ica, a quem foram aplicados dois instrumentos para avaliar a inclusão educativa e a inserção laboral. Os resultados mostram que há uma relação moderada alta, sendo significativa, entre as variáveis inclusão educativa e inserção laboral de pessoas afetadas em seu potencial auditivo. Conclui-se que a inclusão educativa está significativamente relacionada ao aspecto laboral de pessoas com a audição diminuída em Chincha Alta durante o ano de 2019.
Palavras-chave:

Inserção laboral, inclusão educativa, deficiência auditiva, habilitação laboral.

\section{Datos de los autores}

Shirley Mirella Vásquez León Doctora en educación por la Universidad Cesar Vallejo. Trabaja en la Universidad Femenina Sagrado Corazón UNIFÉ. Docente en la especialidad de Matemática.

Edward Faustino Loayza Maturrano es docente e investigador en educación, lingüística, literatura. Magister en Educación por la Universidad Nacional de Educación "Enrique Guzmán y Valle”. 


\section{Introducción}

Un problema que no podemos soslayar en nuestra realidad peruana es la desigualdad debido a las grandes brechas de pobreza aún existentes entre los distintos sectores de la población. Esta situación provoca exclusión y discriminación; y ello, en mayor medida, en aquella población que tiene algún tipo de dificultad o limitación permanente ya sea ésta de tipo visual, auditivo, visoauditivo, motor, cognitivo, etc. (INEI, 2017: 687). La discriminación hacia personas con discapacidad es un problema que el Estado y la sociedad están trabajando para disminuirla con medidas legales que establecen cuotas de inclusión en los sectores educativo y laboral, no obstante, lo iniciado, una parte de la población del país se encuentra sufriendo las consecuencias de este fenómeno.

Los ratios de inclusión que se han establecidos en las instituciones educativas de educación regular de $3 \%$ y $5 \%$ según sea de índole privado o público respectivamente, es una forma de reconocer socialmente que la población con discapacidad es capaz de desarrollarse en todos los contextos que la sociedad posee, es respetar su derecho inalienable a la educación y es terminar con la postergación que durante tanto tiempo ha venido ocurriendo. Cuando una persona se encuentra limitada es la sociedad quien cierra más puertas a este individuo, tachando su condición y haciéndolo incapaz de sostenerse por sus medios, y que siempre están recibiendo ayuda; esta forma de pensar es la que no permite que se incluyan a las personas con limitaciones dentro de los procesos educativos; sin embargo, es necesario que el estado y la sociedad permiten el avance de estas personas, potenciando las habilidades que se destaquen para que así aproveche su talento y pueda mirarse como una persona íntegra que conforma a la sociedad (Ley 27337, 2000:4).

En cuanto a la variedad de opiniones que existe acerca de la discapacidad, pues existen diferentes grupos sociales que realizan campañas y activismo social con el fin de evidenciar las diferencias, grupos que se trata de minimizar en la educación actual. La sociedad y el Estado tienen el deber de velar por una educación de carácter inclusivo que satisfaga las necesidades de las personas discapacitadas y puedan integrarse e incluirse en el sistema educativo de modalidad básica regular y no sólo permitirles una educación en la modalidad básica especial (Ley 28044, 2003). En tal sentido, es obligación de los educadores contribuir al desarrollo de la igualdad, mostrando a cada estudiante que todos tienen los mismos derechos, sin discriminar la condición de otros, y es en ese entonces que comienzan los procesos de inclusión, desde la escuela, para que en el futuro cercano la persona con discapacidad pueda gozar de la inserción y el derecho al trabajo, a ser productivo y valerse por sus medios, es aquí donde se encuentran vinculadas las variables de este estudio: inclusión educativa e inserción laboral.

Estas variables se encuentran vinculadas causalmente, debido a que existe la necesidad de incluir educativa y laboralmente a los individuos con problemas de discapacidad; es éste el motivo que justifica el abordaje de estudios que contribuyan con la resolución de esta problemática; así, se ha tomado como referencia a los jóvenes con problemas de audición del distrito de Chincha Alta ubicado geográficamente dentro de la provincia de Chincha en el departamento de Ica.

El abordaje científico de la discapacidad en el ámbito laboral y educativo no es nuevo. Delmastro y De Montes (2015), examina lo concerniente a la inserción laboral de sujetos con disminución de facultades de ordenanza cognitiva incapacitante para lo cual se realizó talleres, y se obtuvo una conclusión detallada, donde se nota que en las empresas familiares se tiene mejor acogida a las persona con discapacidad, ya que estos tienen el sentido de protec- 
ción de la persona que necesita de ella, mientras que en otras áreas laborales la inserción no resulta muy favorable, puesto que la mayor parte de los empleados tienden a rechazar a los individuos con discapacidad. En otros estudios, se evidencia en el mercado laboral no se les da oportunidades a los sujetos con discapacidad, ya que estos tienen un perfil del empleado predispuestos y estas personas no llenan los requisitos que el cargo necesita, por más que esta persona se encuentre capacitado, porque tiene la especialización necesaria. Los centros educativos que ya se encuentran en procesos de inclusión educativa deben estrechar lazos con el mercado laboral para que las inserciones tengan el mismo efecto que en lo educativo (Vidal y Cornejo, 2012).

En tal sentido, Lazo (2016) concluye que no se hallan mecanismos que faciliten a los ciudadanos con facultades disminuidas donde se puedan incluir y sean empleados a pesar de su condición, no obstante, existen reglamentos que obligan a las empresas a emplear a estos sujetos, pero no hay respeto por parte de las empresas de estas normas, y el estado no se encarga de supervisar, lo que limita a los sujetos a ser productivos.

En Colombia se han realizado estudios referentes a las regulaciones en el tema del trabajo y en el tema de aquellos que tengan algún problema físico donde se estima que la acción de incorporar a los individuos con limitaciones en el mercado laboral es una tarea que requiere de esfuerzos, ya que dentro de las empresas que los contraten tiene que existir la igualdad y las condiciones que les permitan trabajar de forma eficiente y dándoles el derecho que les corresponde (Garavito, 2014). Asimismo, se pudo contemplar como en los sitios de trabajo que insertan a las personas con discapacidad que existe la exclusión por parte de otros empleados, y que no les colaboran o simplemente no les facilitan el trabajo, complicándoles sus tareas (Cendreno, 2017).

En vista a lo observado se evidencia que en el nivel internacional se busca dar un tratamiento social a la persona que tiene alguna capacidad disminuida en el plano profesional; es importante destacar que existen inconvenientes que engloban las destrezas físicas, la economía y lo social, ya que éstas siempre se encuentran con alguna limitación que les impide desempeñarse plenamente pese a que sus esfuerzos siempre sea el doble. Acerca de la predisposición del afectado Lidón (2013), describe en su publicación sobre los derechos que tienen los individuos con discapacidad; cómo estas personas son parte de la sociedad por lo que es necesario que se les permita ser autónomos, sintiéndose normales, aunque tengan limitaciones que otros no padecen, estos les permitirá desarrollarse plenamente, y lo mejor que se puede hacer es que se reconozcan estos derechos.

Al respecto hay estudios nacionales como el de Cortez (2015), quien en su investigación observa las normas legales que en el Perú está aplicando cuáles no. Muestra su acuerdo con apoyar la empleabilidad de los individuos con problemas físicos o mentales. Concluyó con un nivel de significatividad ( $r s=0,643, p<0,05$ ), entre el cumplimiento de las normas peruanas y el empleo de discapacitados; asimismo, la disposición de la sociedad en el fomento del trabajo en individuos con discapacidad, señalando que para las personas con discapacidad puedan optar por un trabajo digno debe tenerse en cuenta cuál es el puesto que más se ajusta a su limitación.

Por otro lado, acerca de la variable inclusión educativa existen estudios internacionales como el de Walsh (2018) quien investigó acerca del procedimiento para incorporar alumnos con discapacidades disminuidas en el salón de clases generales. Señaló que se pueden utilizar diversas estrategias para implementar la enseñanza en pequeños que se encuentran limitados por alguna discapacidad, pero con la colaboración de todos los estudiantes, para que éstos incluyan al niño, siendo colaborativos entre todos, que les brinden el apoyo necesario, así como 
en diferentes oportunidades individualizar la atención docente-estudiante, para que pueda sentir que no es diferente al resto, también se les debe fijar un patrón constante, para que el niño se sienta en confianza y su desenvolvimiento sea más rápido y se adapte a la nueva circunstancia por la que pasa.

En referencia a ello, Murphy (2014) estudia la reacción de los profesores al recibir estudiantes con necesidades distintas al resto. Éste destaca las reacciones de los profesores que no están capacitados para recibir dentro del aula a alumnos que necesitan de una atención especial; manifiesta que éstos son los primeros en rechazar la presencia de estos pequeños, haciéndolos sentir menospreciados y fuera de lugar, por tanto, es necesario que se siga profundizando en el tema, y capacitar a los profesores para que la problemática no sea concurrente; el estudio ha demostrado que los profesores han sido incapaces de tomar las recomendaciones que se han hecho en estudios anteriores, pudiendo facilitar su labor. King y Edmunds (2001) han manifestado que los directivos deben tener presente las opiniones de los docentes, ya que son estos los encargados de proceso de inclusión al estudiante.

La opinión y creencias de los educadores sobre lo estudiado, es dejar que estos expresen cuáles han sido las necesidades que perciben en el aula, ya que se encargan del bienestar propio y de sus estudiantes. Según Saleem (2017) quien basó su estudio en las reacciones de los maestros hacia la inclusión de infantes que necesitan cuidado especial en los centros ordinarios de Jordania; pudo notar que los docentes han reaccionado de forma positiva al saber que tienen estudiantes con limitaciones por discapacidad, y muestran que el estudiante es normal, incluyéndolos inmediatamente, así como valora sus capacidades intelectuales, y demostrándoles afecto; no obstante, a ello, también se reconoció que muchos de los docentes no se encuentran bien preparados para la atención que estos estudiantes requieren, así estén llevando a cabalidad el proceso de incluirlos.

Para entender mejor sobre la discapacidad y la educación, es necesario que se sustente en la teoría del crecimiento del conocimiento, la teoría del conocimiento sociocultural, expuesta por Vygotsky, ya que este se encargó de estudiar detalladamente las características del aprendizaje en los niños, en los considerados normales como en aquellos que padecían de alguna limitación. Asimismo, Vygotsky (1982) expresa que el lenguaje es la vía de comunicación y aprendizaje de todo ser humano, para los niños que se encuentran limitados auditivamente, se necesitó utilizar el método de lenguaje por señas, estableciendo patrones que le ayudasen a comprender las palabras y facilitando el aprendizaje que estos puedan obtener.

Es necesario destacar que a través del lenguaje es que los individuos expresan emociones y logran comunicarse con la sociedad, dicho esto, es importante darle valor a las limitaciones que tiene un niño cuando se encuentra en desventaja por no poder expresar de forma oral lo que siente, y se necesita de personas que estén dispuestos a incluirlos en la sociedad, y que no se tolere la discriminación.

De igual manera, en lo que respecta al plano de los derechos humanos, se pudo concluir que se comete un grave error cuando hay prácticas de exclusión de personas limitadas, dicho comportamiento no debe permitirse (Nussbaum, 2007, p.110). Se considera que la sociedad ha avanzado a pasos agigantados, ya que cada vez más se logra detectar la inclusión de personas con alguna discapacidad, mientras más transcurre el tiempo, este proceso se siente natural. La sociedad también ha entendido que muchos de los defectos congénitos se pasan genéticamente, lo que ha colaborado con el entendimiento y la aceptación en la sociedad. Es por ello que, la aceptación ha sido cada vez mayor, porque el entendimiento de los individuos ha 
evolucionado, y se les otorga los mismos derechos, para que éstos puedan aprovechar al máximo de los beneficios que el resto ya tiene. Luego, ante la ley del Estado y del hombre, cada individuo es igual sin importar las diferencias que se notan entre todos, es por esa razón que el Estado debe regir con igualdad y equidad, y que cada persona debe regirse por las mismas normas. Ello conlleva a que en la educación se incluyan a todos los estudiantes sin importar su condición, para que la inserción profesional sea más fácil.

Como antecedente nacional, se tiene que, en el Perú se ha dado a conocer el Proyecto Educativo Nacional al 2021, donde su principal propósito es incluir en la sociedad a cada persona que se considera diferente, y es un acuerdo que la población debe comprometerse a cumplir, para que se tenga igualdad de condiciones en el área educativa. En referencia a lo mencionado, el Instituto Nacional de Estadística e informática del Perú (INEI), ha podido verificar que en la población peruana se encuentra 1.500 .000 de individuos sufriendo de discapacidades, y más del $50 \%$ de ellos son mujeres.

Asimismo, el proceso para incluir en la educación a todos los individuos, la Unesco (citado en Moliner, 2013), expresa que esta es una necesidad que tiene mucho tiempo en el mundo entero, pero que en la actualidad se está tomando más en serio incluir a toda persona que tiene limitantes, y más aún dentro de los centro educativos, ya que es el centro principal del aprendizaje formalizado, fomentando esa cultura en los estudiantes, para que en un futuro no sea visto como un tabú, o un tema más que ocultar al mundo, ya que todos pertenecemos a una sociedad activa, muchas veces se comete el error de menospreciar los talentos de los niños discapacitados, pues muchos de éstos pueden tener una capacidad intelectual mayor que el resto.

El sistema pedagógico actualmente desafía que los niños deben instruirse unidos, sin mantener en cuenta discrepancias personales y de su ambiente; de esta forma se debe conceder paralelismo de oportunidades. En tal sentido, se debe determinar circunstancias y contextos, para que los adolescentes puedan incorporarse a un amaestramiento característico sin impedimentos, no obstante, a ello, se implementarán los contextos pertinentes para lograr el objetivo, los profesores deben instruirse por medio de capacitaciones intensivas referentes a la lengua de señas peruana. Como es de saber la problemática auditiva perjudica la obtención y progreso de la expresión en los infantes.

De otra parte, en este estudio las variables a correlacionar: inclusión educativa e inserción laboral requieren por su parte una definición conceptual y una explicación de sus dimensiones que las operacionalizan. Primero, la variable inclusión educativa, es aquel proceso que debe ser promovido para que cada estudiante se acepte tal cual como es, teniendo o no limitaciones, haciéndoles saber que tienen derechos ante el mundo desde el momento que conforman parte de la sociedad y que son igualitarias al resto. (UNESCO, 2008, p.7). La variable inclusión educativa es muy compleja ya que el concepto inclusión tiene un valor de contenido rígido, no solo se refiere al hecho de proporcionar educación a las personas que tienen alguna discapacidad, en igualdad de condiciones, es también una concepción de índole sociopolítica (Parrilla, 2002). Pero en la actualidad se destaca el significado sobre cómo identificar y clasificar a los niños y niñas que necesitan ser incluido por tener dificultades ya que según Pérez (2011), ha habido clasificaciones de carácter ambiguo aun en profesionales (médicos y educadores). También se define a la inclusión educativa como un proceso de eliminación de barreras que tiene que estrechar la relación con la asistencia, incorporación y alcance de los alumnos (Echeita y Ainscow, 2010).

En cuanto a las dimensiones se menciona: dimensión 1: Presencia, se encuentra vinculada con la preservación del nivel del alumno, sin descuidar las clases. (Unesco, 2008, p.7). Esta 
dimensión está íntimamente ligada al hecho de la asistencia a clase, asistencia que debe ser regular, lo cual probaría que los estudiantes incluidos no temen a los demás o no temen a su entorno social, por otro lado se da por parte del estado la universalización de la matrícula, lo cual hace que los niños incluidos permanezcan al menos un periodo de las clases, esta dimensión está ligada a la permanencia en la comunidad, niños o adolescentes incluidos demostrando que en las escuelas inclusivas existe un alto compromiso con la matricula del estudiantado, lo que permite observar el hecho de que un niño pueda ser incluido o excluido de una escuela regular, lo cual es un indicador (Giné y Font, 2007). Con respecto a la segunda dimensión: la participación, alude a que toda ley o contenido que presente el método pedagógico, debe considerarse que a todo individuo con problemas físicos o mentales se le garantice un trato acorde y con semejanza a otras personas (Unesco, 2008, p.7), esta dimensión tiene la finalidad de enfocar al estudiante incluido en pleno desarrollo de sus actividades, ya que es diferente que sea aceptado a que sea incluido, si es aceptado solo podría permanecer a un lado ignorado, pero si participa en realidad se ha incluido en el grupo social. La tarea entonces en torno a esta dimensión es eliminar barreras, por lo que hay que observar indicadores tales como infraestructura, rigidez curricular, ausentismo, pobreza, entre otros (Ainscow y Miles, 2001). Con respecto a la tercera subvariable denominada logros, es relativo al estudiante adopte una postura crítica de sus propios alcances, obtenidos a partir de normas determinantes del sistema educativo (Unesco, 2008, p.7). Los estudiantes incluidos deben alcanzar, en igualdad de oportunidades, en lo concerniente a los logros también ingresa el tratamiento de la denominada “inclusión en los aprendizajes" (Blanco, 2011; Marchesi, 2014).

Sobre la variable inserción laboral, es aquella oportunidad que se le debe brindar a las personas que tienen limitaciones, aunque su perfil muchas veces no cumpla con las expectativas del puesto, tarea que le corresponde al estado, fijar normas de inclusión educativa para que estos individuos se preparen y puedan tener una oportunidad laboral (Álvarez, 2017, p. 27). La inserción laboral se caracteriza por incluir en un puesto a un trabajador, en este caso una persona con necesidades especiales, por ello cuando se aplica el paradigma del Empleo con Apoyo se constituye en medio valido para la comunidad internacional para estar al pendiente con los potenciales trabajadores con carencias particulares (Vidal y Cornejo, 2012). Del mismo modo se tiene que la definición de inserción laboral se centra en el hecho de poder engendrar valor al trabajo que se realiza, valor de alta profesionalidad, teniendo como punto central la diversidad y en aspectos claves que las oficinas de recursos humanos deben considerar para que exista un desempeño eficaz en el puesto de trabajo (Forcada, 2015).

En tanto a la variable estudiada, se desglosan una serie de dimensiones, las cuales se evidencian a continuación, dimensión 1: Barreras de accesibilidad. Consideradas como las trabas al individuo con problemas físicos y mentales desempeño un cargo en cualquier organización bien sea pública o privada. (Álvarez, 2017: 32). La subvariable segunda: Integración laboral, se refiere al grado aceptado por el individuo con problemas físicos o mentales, comprendiendo así su problema para ejercer sus funciones dentro del área laboral. (Álvarez, 2017: 33). Dimensión 3: Habilitación, Alude al apoyo a aquel individuo que lo necesite en vista de su condición física o mental, ayudándolo así a que pueda adquirir de una manera eficaz y practica estrategias o habilidades para laborar sin problemas (Álvarez, 2017: 31).

Es por esa razón que se llegó a formular la siguiente interrogante: ¿Cuál es la relación entre los mecanismos de aceptación laboral y la pragmática inclusiva dirigidos a los jóvenes que tiene funciones auditivas disminuidas en el distrito de Chincha Alta el 2019?; asimismo, se formularon las interrogantes para precisar la cuestión: ¿Cuál es la relación entre la subvariable barreras de accesibilidad y la inclusión educativa en Chincha Alta el 2019?, ¿Cuál es la relación entre las entre 
la colocación laboral y la inclusión educativa de los jóvenes con disminución de funciones auditivas en Chincha Alta el 2019?, ¿Cuál es la relación entre las entre la habilitación y la inclusión educativa de jóvenes con discapacidad auditiva en Chincha Alta el 2019?; lográndose extraer datos a partir de la aplicación sistemática de cuestionarios que contendrán información sobre la inclusión en la educación, y el recibimiento de personas limitadas por discapacidades en áreas laborales, y que contemplarán normas suscritas en la ley, así como también detalles técnicos, todos esos datos contenidos en los cuestionarios serán moldeados a la discapacidad auditiva.

Los resultados de la indagación tuvieron una finalidad fundamental, la de medir el grado de asociación de la subvariable referida al aspecto laboral y en materia educativa de post adolescentes que presentan discapacidad auditiva en Chincha Alta el 2019; y específicamente medir dicha asociación entre las dimensiones de la variable del aspecto laboral y la referida a Educación, teniendo como unidad de análisis la población de personas post adolescentes con disfunciones auditivas en Chincha Alta el 2019.

De igual manera se planteó una hipótesis general referida a calcular el grado de significancia de la relación entre variables, las mismas que han sido medidas en personas con problemas auditivos en Chincha Alta el 2019, además para completar el sistema de hipótesis y respondiendo a los objetivos también se calculó el nivel de significancia de la relación entre las especificidades de la variable respecto a lo laboral y la inclusión educativa en postadolescentes con dificultades de audición auditiva en el distrito de Chincha Alta el 2019.

Los estudios examinados hasta aquí si bien relacionan las variables inserción laboral e inclusión educativa, no operacionalizan de modo pertinente estas variables lo cual constituye una debilidad; condición que justifica el aporte del presente estudio. Por ello, esta investigación plantea una propuesta conceptual y metodológica inédita y pertinente sobre los indicadores a correlacionar. Dilucidar los aspectos a relacionar dentro de cada variable (intravariable) y determinar la relación que existe entre cada uno de los aspectos e indicadores de variable causal con los aspectos e indicadores de la variable efecto (intervariable) es relevante.

Asimismo, la población estudiada que posee una discapacidad de tipo auditiva no queda limitada, en lo absoluto, de sus facultades mentales y físicas, por lo que antes de hablar de "inclusión educativa" hay que reconocer que existe el problema de "aceptación educativa" de parte de las distintas instituciones de la sociedad (familia, escuela, economía, política, etc.), lo cual configura un problema cultural. La pérdida de un sentido como la audición supone por un proceso de reequilibración psicobiológica la afinación o mayor desarrollo de los otros sentidos. (Pernía, 2019) Entonces surgen individuos con nuevas habilidades y sentidos mejor dotados; por eso quienes poseen discapacidad auditiva se les denomina personas con habilidades diferentes. La denominación de discapacidad es una estigmatización efectuada por el contexto social; así las personas con discapacidad auditiva severa poseen una condición antes que una discapacidad. Desde este enfoque social se sostiene que las personas con discapacidad deben ser incluidas en las escuelas y en el mundo laboral. En tal sentido, las escuelas se deben adaptar a ellos y no ellos a las escuelas. Igualmente, en los trabajos se deben dar las condiciones para que ellos logren ser empleados, incluidos y valorados.

La educación en tanto sistema social es definida culturalmente, por lo que la exclusión es una variable sociológica a la cual se someten los individuos como parte de su integración. La sociedad está diseñada para las personas con características comunes y generales, cuya inserción social es llamada normal, sin embargo, las personas con sordera constituyen una población minoritaria que requiere una adaptación social distinta. Ciertamente, logran adap- 
tarse, pero tienen que superar mayores dificultades. El presente estudio si bien se centra en establecer correlaciones entre inserción laboral e inclusión educativa también pretende servir de estrategia de intervención (política y social) para la inclusión educativa e inserción laboral de las poblaciones minoritarias, a veces excluidas, a veces discriminadas como es el caso de la población sorda. En ese sentido, los instrumentos elaborados como unidades de análisis permiten observar por medio de una especificación de las unidades de observación las condiciones y categorización de la ocurrencia del fenómeno.

\section{Metodología}

Diseño: El diseño de la investigación es correlacional de tipo transversal, a que su propósito fue determinar la asociación entre las variables inclusión educativa e inserción laboral de jóvenes con discapacidad auditiva en el mismo grupo de individuos.

Participantes: Se consideraron un total de 106 jóvenes con discapacidad auditiva del distrito de Chincha Alta cuyas edades fluctuaban entre los 16 y 44 años, 42 de los cuales fueron de sexo femenino y 64 del masculino. La selección de los participantes fue favorecida por la disponibilidad de acceso a la muestra. La población de jóvenes con discapacidad auditiva del distrito de Chincha Alta es de 151 según datos obtenidos en el último censo de población y vivienda del Perú referido a los resultados estadísticos correspondientes al distrito de Chincha Alta del departamento de Ica (INEI, 2017: 690).

Instrumentos: En esta investigación fueron utilizados dos instrumentos. En primer lugar, el Cuestionario sobre Inclusión Educativa elaborado por Shirley Mirella Vásquez León el 2019 cuyo objetivo es conocer el grado de inclusión educativa que presentan los individuos con discapacidad auditiva. Este es un cuestionario de aplicación individual de veinte minutos de duración. Consta de 24 ítems con tres alternativas de respuesta de opción múltiple de tipo Likert: (1) Nunca (2) A veces (3) Siempre. Asimismo, la escala está conformada por tres dimensiones y los baremos siguientes: Alto [33-48]- Regular [17-32]- Bajo [0-16]. El coeficiente de confiabilidad del intrumento es el alfa de Cronbach obtenido fue de 0.881 lo cual nos señala el nivel de fiabilidad que presenta el instrumento.

En segundo lugar, el Cuestionario sobre Inserción Laboral elaborado por Shirley Mirella Vásquez León el 2019 cuyo objetivo es conocer el grado de inserción laboral que presentan los individuos con discapacidad auditiva. Este es un cuestionario de aplicación individual de veinte minutos de duración. Consta de 24 ítems con tres alternativas de respuesta de opción múltiple de tipo Likert: (1) Nunca (2) A veces (3) Siempre. Asimismo, la escala está conformada por tres dimensiones y los baremos siguientes: Alto [33-48]- Regular [17-32]- Bajo [0-16]. El coeficiente de confiabilidad del instrumento es el alfa de Cronbach obtenido fue de 0.886 lo cual nos señala el nivel de fiabilidad que presenta el instrumento.

Procedimientos: La presente investigación necesitó acercarse a las distintas instituciones locales (instituciones educativas, centros comerciales, empresas familiares, etc.) donde estudian y trabajan los jóvenes con discapacidad auditiva del distrito de Chincha Alta. Se aplicaron los cuestionarios en diferentes momentos a las mismas personas. Luego de la aplicación se les informó a los jóvenes sobre el propósito de la investigación con el fin que no se produzca ningún efecto halo que distorsione las respuestas previamente. Posterior de la aplicación los datos fueron ingresados al programa estadístico SPSS versión 23, en donde se hicieron los respectivos análisis descriptivos e inferenciales. 


\section{Resultados}

\section{Resultados descriptivos}

Se observa que el $5,7 \%$ (6) de los sujetos de la muestra se ubican a nivel regular con respecto a la inserción laboral, el 61,3\% (65) se perciben bajo, mientras que el 33\% (35) se coloca en muy bajo y el o\% (o) se categoriza como alto.

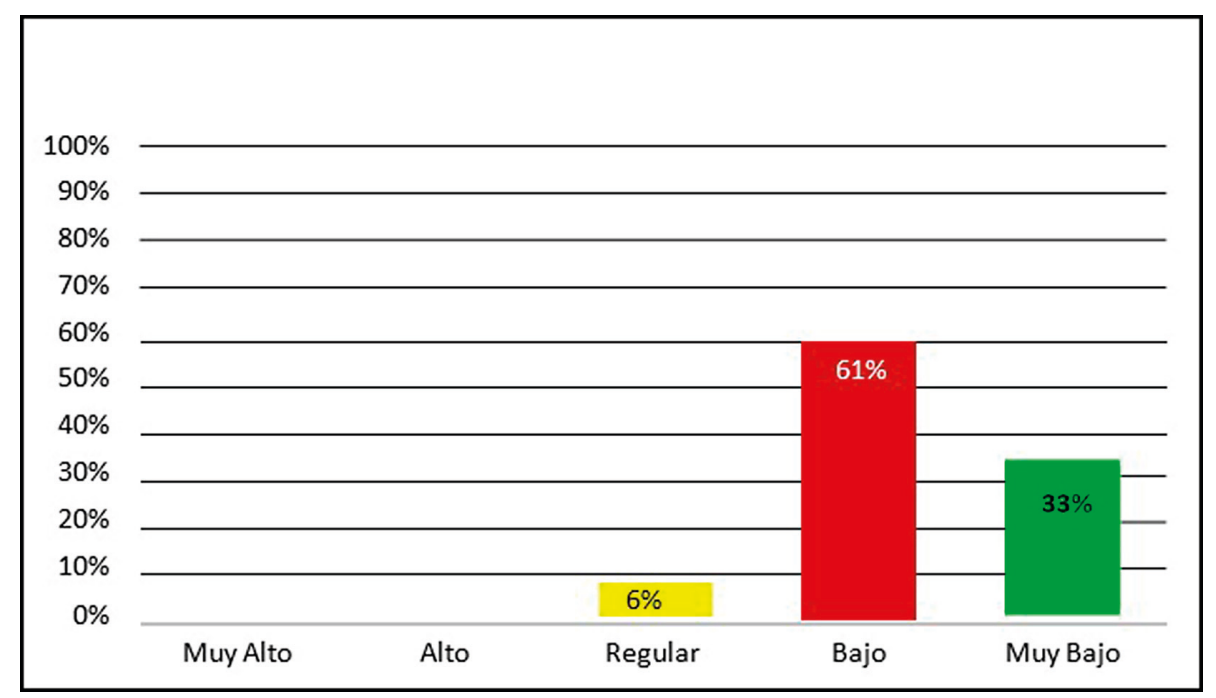

Figura 1. Distribución de los niveles de Inserción Laboral

Se evidencia que el o\% (o) de los sometidos al cuestionario preparado se localiza en un rango alto en torno a la inclusión educativa, el o\% (o) está en un nivel regular, el 61,3\% (65) se estima bajo y lo más elevado correspondiente al 38,7\% (41) se posicionan en la categoría muy baja.

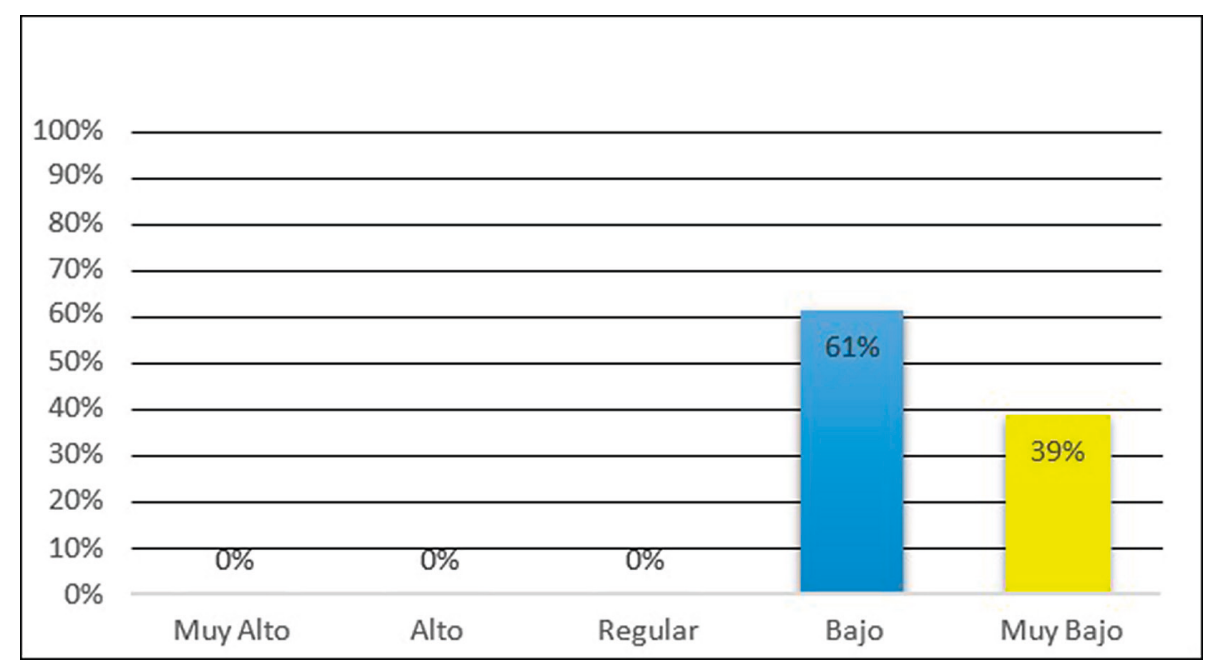

Figura 2. Distribución de los niveles de inclusión educativa. 


\section{Resultados inferenciales}

$\mathrm{Hi}=$ La inclusión educativa se relaciona significativamente con la inserción laboral de jóvenes con discapacidad auditiva en Chincha Alta en el 2019.

Ho= La inclusión educativa no se relaciona significativamente con la inserción laboral de jóvenes con discapacidad auditiva en Chincha Alta en el 2019

Tabla 1

Correlación entre la Inserción Laboral y la Inclusión Educativa.

\begin{tabular}{|c|c|c|c|c|}
\hline & & & $\begin{array}{r}\text { Inserción } \\
\text { Laboral }\end{array}$ & $\begin{array}{l}\text { Inclusión } \\
\text { Educativa }\end{array}$ \\
\hline \multirow{6}{*}{$\begin{array}{l}\text { Rho de } \\
\text { Spearman }\end{array}$} & Inserción Laboral & $\begin{array}{c}\text { Coeficiente de } \\
\text { correlación }\end{array}$ & 1,000 &, $639^{\star *}$ \\
\hline & & Sig. (bilateral) & . & , ০০০ \\
\hline & & $\mathrm{N}$ & 106 & 106 \\
\hline & $\begin{array}{l}\text { Inclusión } \\
\text { Educativa }\end{array}$ & $\begin{array}{l}\text { Coeficiente de } \\
\text { correlación }\end{array}$ &, $639^{* *}$ & 1,000 \\
\hline & & Sig. (bilateral) & , ০০০ & . \\
\hline & & $\mathrm{N}$ & 106 & 106 \\
\hline
\end{tabular}

**. La correlación es significativa en el nivel o, o1 (bilateral).

Como $\boldsymbol{p}$ - valor $<\mathbf{0 , 0 1}$ Es rechazada la hipótesis nula y es aceptada la alterna concluyendo que se presenta una correlación positiva entre la inclusión educativa e inserción laboral con un valor $r=0.639$ detectado por la prueba Rho de Spearman.

Hipótesis específica 1

Ho= La inclusión educativa no se relaciona significativamente con accesibilidad de jóvenes con discapacidad auditiva en Chincha Alta en el 2019.

$\mathrm{H}_{1}=\mathrm{La}$ inclusión educativa se relaciona significativamente con accesibilidad de jóvenes con discapacidad auditiva en Chincha Alta en el 2019.

Tabla 2

La inclusión educativa y accesibilidad

\begin{tabular}{|c|c|c|c|c|}
\hline & & & $\begin{array}{l}\text { Inclusión } \\
\text { Educativa }\end{array}$ & accesibilidad \\
\hline $\begin{array}{l}\text { Rho } \\
\text { de }\end{array}$ & Inclusión Educativa & $\begin{array}{l}\text { Coeficiente de } \\
\text { correlación }\end{array}$ & 1,000 &, $524^{\star \star}$ \\
\hline \multirow[t]{5}{*}{ Spearman } & & Sig. (bilateral) & . & , ০০० \\
\hline & & $\mathrm{N}$ & 106 & 106 \\
\hline & Accesibilidad & $\begin{array}{l}\text { Coeficiente de } \\
\text { correlación }\end{array}$ &, $524^{\star *}$ & 1,000 \\
\hline & & Sig. (bilateral) & , ০০o & . \\
\hline & & $\mathrm{N}$ & 106 & 106 \\
\hline
\end{tabular}

**. La correlación es significativa en el nivel o,01 (bilateral). 
Como $\boldsymbol{p}$ - valor $<\mathbf{0 , 0 1}$ Es rechazada la hipótesis nula y es aceptada la alterna concluyendo que se presenta una correlación positiva entre la inclusión educativa y accesibilidad con un valor $r=0.524$ detectado por la prueba Rho de Spearman.

Hipótesis específica 2

Ho= La inclusión educativa no se relaciona significativamente con la integración laboral de jóvenes con discapacidad auditiva en Chincha Alta en el 2019

$\mathrm{H}_{2}=$ La inclusión educativa se relaciona significativamente con la integración laboral de jóvenes con discapacidad auditiva en Chincha Alta en el 2019

Tabla 3

La Inclusión Educativa y la Integración Laboral de Jóvenes con Discapacidad Auditiva en Chincha Alta en el 2019

\begin{tabular}{|c|c|c|c|c|}
\hline & & & $\begin{array}{l}\text { Inclusión } \\
\text { Educativa }\end{array}$ & $\begin{array}{c}\text { Integración } \\
\text { Laboral }\end{array}$ \\
\hline \multirow{6}{*}{$\begin{array}{l}\text { Rho } \\
\text { de } \\
\text { Spearman }\end{array}$} & \multirow[t]{3}{*}{$\begin{array}{l}\text { Inclusión } \\
\text { Educativa }\end{array}$} & $\begin{array}{l}\text { Coeficiente de } \\
\text { correlación }\end{array}$ & 1,000 & $491^{\star *}$ \\
\hline & & Sig. (bilateral) & . & , , o० \\
\hline & & $\mathrm{N}$ & 106 & 106 \\
\hline & \multirow[t]{3}{*}{$\begin{array}{l}\text { Integración } \\
\text { Laboral }\end{array}$} & $\begin{array}{l}\text { Coeficiente de } \\
\text { correlación }\end{array}$ &, $491^{\star *}$ & 1,000 \\
\hline & & Sig. (bilateral) & , ০০o & . \\
\hline & & $\mathrm{N}$ & 106 & 106 \\
\hline
\end{tabular}

**. La correlación es significativa en el nivel o,01

(bilateral).

Como $\boldsymbol{p}$ - valor $<\mathbf{0 , 0 1}$ Es rechazada la hipótesis nula y es aceptada la alterna concluyendo que se presenta una correlación positiva entre la inclusión educativa e Integración Laboral con un valor $r=0.491$ detectado por la prueba Rho de Spearman.

Hipótesis específica 3

Ho= La inclusión educativa no se relaciona significativamente con la habilitación de jóvenes con discapacidad auditiva en Chincha Alta en el 2019.

$\mathrm{H}_{1}=\mathrm{La}$ inclusión educativa se relaciona significativamente con la habilitación de jóvenes con discapacidad auditiva en Chincha Alta en el 2019. 


\section{Tabla 4 \\ La inclusión educativa y la habilitación de jóvenes con discapacidad auditiva en Chincha Alta en el 2019}

\begin{tabular}{lllcc}
\hline & & $\begin{array}{c}\text { Inclusión } \\
\text { Educativa }\end{array}$ & Habilitación \\
\hline $\begin{array}{l}\text { Rho } \\
\text { de } \\
\text { Spearman }\end{array}$ & Inclusión Educativa & Coeficiente de correlación & 1,000 &, $554^{* *}$ \\
& & Sig. (bilateral) &. &, 000 \\
& $N$ & 106 & 106 \\
& Habilitación & Coeficiente de correlación &, $554^{* *}$ & 1,000 \\
& & Sig. (bilateral) &, 000 &. \\
& & $N$ & 106 & 106 \\
\hline
\end{tabular}

**. La correlación es significativa en el nivel o,o1 (bilateral).

Como $\boldsymbol{p}$ - valor $<\mathbf{0 , 0 1}$ Es rechazada la hipótesis nula y es aceptada la alterna concluyendo que se presenta una correlación positiva entre la inclusión educativa y la habilitación laboral con un valor $\mathrm{r}=0.554$ detectado por la prueba Rho de Spearman.

Los resultados, asimismo, demuestran no solo las correlaciones positivas entre las variables también remarcan la validez y pertinencia de las subvariables, los indicadores y ítems planteados en los instrumentos de observación; lo cual es una validación de facto de estos criterios que pueden considerarse como aspectos específicos para el fomento de equidad entre las poblaciones: la mayoritaria (sin discapacidad) y las poblaciones minoritarias (con discapacidad) como es el caso de la población con déficit de audición del país.

Por tanto, el trabajo de investigación consigue probar que la variable inclusión educativa supone tres dimensiones: la presencia en la escuela (accesibilidad y tiempo de presencialidad), participación en la escuela (atención de necesidades, apoyo y respeto a sus decisiones), logros del estudiante (competencias de aprendizaje esperados, integración y socialización con sus pares oyentes).

Asimismo, se validan los indicadores dentro de cada dimensión: $1^{\circ}$ Presencia del individuo con discapacidad en el sistema educativo, lo cual se observa a través de dos indicadores: a) acceso a la escuela del estudiante con discapacidad auditiva, esto es, facilidades y condiciones para su ingreso; b) tiempo de permanencia del estudiante en la escuela, es decir, tasa de repitencia, tiempo de permanencia en primaria y secundaria, años que le toma para terminar cada nivel.

$2^{\circ}$ Participación de la persona con discapacidad auditiva se evidencia mediante tres indicadores: a) atención de las necesidades de acuerdo a su discapacidad por parte de la escuela; b) atención y respeto a sus decisiones sobre su diario vivir dentro del entorno escolar; c) respeto y escucha de sus opiniones sobre el funcionamiento de la escuela.

$3^{\circ}$ Logro del estudiante con sordera se demuestra a través de dos indicadores: a) logro de aprendizajes previstos, es decir, la escuela empleaba actividades de aprendizaje para sordos o existía adaptaciones pertinentes; b) desarrollo de socialización eficaz entre él y sus compañeros oyentes mediante participaciones en actuaciones u otras actividades públicas y su nivel de integración en el grupo. 
Por otro lado, en la variable inserción laboral, según el presente estudio se puede evidenciar esta condición a través de tres dimensiones: barreras de accesibilidad (obstáculos en los mecanismos de selección, existencia de plazas especiales), integración laboral (regulación, existencia de políticas, proceso de inducción), habilitación (condiciones sociales, condiciones propiciadas por el estado).

$1^{\circ}$ Barreras de accesibilidad laboral se evidencia mediante dos indicadores: a) mecanismos de selección de personal, lo que implica el grado de consideración de las personas con discapacidad en las convocatorias de personal; así como si durante el desempeño laboral se toman en consideración las condiciones especiales de las personas con discapacidad: el respeto del puntaje especial asignado a personas con discapacidad en los procesos de selección, el reclutamiento de personal le da prioridad a las personas con discapacidad. b) existencia de plazas especiales para discapacitados, lo que supone convocatorias de plazas para personas con discapacidad, reserva de cierta cantidad de vacantes para personas con discapacidad.

$2^{\circ}$ Integración laboral es una dimensión de la variable que se observa a través de tres indicadores: a) regulación laboral, el cual permite conocer si los reglamentos en el centro laboral regulan de manera especial las condiciones laborales de las personas con discapacidad y si se tienen consideraciones especiales que faciliten el desarrollo de sus labores; b) existencia de políticas, para conocer si se propician e implementan políticas de capacitación laboral al personal para la correcta integración laboral de las personas con discapacidad; c) proceso de inducción, con el fin de determinar si la persona con discapacidad es acompañada por un tutor o entrenador laboral especializado al inicio del ejercicio de su contrato laboral, o si se le brinda tecnología adecuada para que pueda laborar eficazmente.

$3^{\circ}$ Habilitación laboral, segunda dimensión que se comprueba mediante dos indicadores: a) condiciones sociales, para entender si a las personas con discapacidad se les otorga la ventaja social en el acceso al puesto de trabajo, que según ley le corresponde; o si también para las personas con discapacidad se cumplen con el horario establecido dentro de la empresa. b) condiciones propiciadas por el Estado, es decir, si los planes o programas de protección social que implementa el Estado para las personas con discapacidad al momento de incorporarse al campo laboral son eficientes; o si el Ministerio del Trabajo participa en el acompañamiento de inserción laboral de las personas con discapacidad.

En los cuestionarios aplicados a los jóvenes con discapacidad auditiva en la ciudad de Chincha Alta se ha evidenciado que ellos mayoritariamente trabajan; no obstante, los instrumentos no registran a qué clase de trabajo se dedican. Un dato importante para valorar cómo el estatus socioeconómico permite la inclusión educativa e inserción laboral. Los discapacitados como población minoritaria también tienen aspiraciones de estudiar una carrera, tener un trabajo digno, formar familia y ser ellos el sustento de su hogar; sin embargo, se ven impedidos o limitados por la escasez de oportunidades, la falta de respeto a su condición y a la transgresión de sus derechos. Así los que logran prepararse (ser profesionales) son los que gozan de una economía elevada y pueden estudiar con el acompañamiento de un traductor.

En este sentido, las personas con discapacidad auditiva tienen que enfrentar una realidad dura, debido a que no cuentan con los medios económicos y porque el apoyo del Estado es insuficiente. Generalmente se emplean como personal de limpieza, ayudantes en tienda de abarrotes, juguerías, estibadores en el terminal pesquero; es decir, en trabajos manuales. Esto significa que su expectativa laboral está limitada por las condiciones que la sociedad le 
impone. Las personas con discapacidad auditiva quieren conocer y dominar el mundo lo más pronto posible; sin embargo, cuando se enfrenta a la realidad empieza la frustración por no poder realizarse o desarrollarse. Las empresas se rehúsan a cumplir con lo establecido en la ley; respecto a emplear a estas personas según los ratios establecidos. Una razón para este fenómeno es la barrera lingüística que supone la sordera, y también, al poco conocimiento que se tiene de la comunicación a través de la lengua de señas, la cual es considerada como otro idioma; más aún si no hay suficiente personal traductor en el país, (solo hay 23 traductores certificados).

\section{Discusión}

En este trabajo de investigación se ha podido demostrar el grado asociativo de las variables de estudio referidas al plano laboral en post adolescentes con problemas auditivos en Chincha Alta en el 2019. En tal sentido, como resultado de la indagación, según lo indicado por Unesco (2008), ésta es una necesidad que tiene mucho tiempo en el mundo entero, pero que en la actualidad se está tomando más en serio incluir a toda persona que tiene limitantes, y más aún dentro de los centro educativos, ya que es la raíz del aprendizaje, fomentando de esta forma la cultura del respeto y toma de conciencia entre los estudiantes, con el propósito que no se siga viendo como un tabú, o un tema más que ocultar al mundo, ya que todos pertenecemos a una sociedad activa, y muchas veces se comete el error de menospreciar los talentos de los niños discapacitados, incluso si éstos, en muchos casos, pueden tener una capacidad intelectual mayor que el resto.

Asimismo, Álvarez, (2017) sostuvo que la inserción laboral es aquella oportunidad que se le debe brindar a las personas que tienen limitaciones, aunque su perfil muchas veces no cumpla con las expectativas del puesto, tarea que le corresponde al Estado, fijar normas de inclusión educativa para que estos individuos se preparen y puedan tener una oportunidad laboral. Este progreso relevante está configurado tanto en el estudio descriptivo (tablas y gráficos) como también en el análisis inferencial. Asimismo, siguiendo el proceso investigativo, se aplicó el estadístico denominado Rho de Spearman para conocer la atracción entre ambos elementos de estudio. Los resultados obtenidos según la variable, tomando como referencia su equivalente porcentual, así como sus dimensiones, en este sentido se tiene que el $76 \%$ (78) se coloca en un nivel muy bajo y solo el $1 \%(1)$ se categoriza como alto, indicando ello que el nivel que se alcanza como respuesta de los sujetos entrevistados en referencia la inserción laboral, está en buen nivel ello significa que en el distrito de Chincha Alta durante el año 2019, han dado resultado las políticas inclusivas que han permitido insertar laboralmente a las personas afectadas con problemas de audición.

Igualmente, se utilizó el estadístico de Rho de Spearman para conocer la asociación entre ambos elementos de estudio, corroborándose dicha información, a través de la prueba de hipótesis que evidenció el valor de 0,639 en grado positiva moderada y un ( $p$ ) valor de o,00 <0.05; negándose la hipótesis nula con la aceptación de la hipótesis de investigación. Estos datos confirman que la inclusión educativa se vincula positivamente con la inserción laboral de post adolescentes con problemas auditivos en Chincha Alta en el 2019. Los resultados similares con los obtenidos por Saleem (2017) basó su estudio en los docentes y adolescentes de Jordania. Pudo notar que los docentes han reaccionado de forma positiva al saber que tienen estudiantes con limitaciones por discapacidad, y hacen ver que el estudiante es normal, incluyéndolos inmediatamente, así como valora sus capacidades intelectuales, y demostrándoles afecto; no obstante, a ello, también se reconoció que muchos de los docentes no se encuen- 
tran bien preparados para la atención que estos estudiantes requieren, así estén llevando a cabalidad el proceso de incluirlos. Concluye con un vínculo significativo ( $r s=0,527, p<0,05$ ), que necesitan cuidado especial en los centros educativos ordinarios de Jordania, Igualmente la atención se fundamenta en lo señalado por Unesco (2008) y Álvarez, (2017).

\section{Conclusiones}

El análisis de los resultados y las correlaciones entre las variables permiten establecer las siguientes conclusiones:

Primera conclusión: La hipótesis principal de la investigación estima que H: La inclusión educativa se relaciona significativamente con la variable tratada respecto al aspecto laboral de las personas post adolescentes con problemas de inserción laboral en Chincha Alta en el 2019, y considerando que $\boldsymbol{p}$ - valor $<0,01$ en consecuencia no se ha tenido en cuenta la hipótesis nula y se aceptó la hipótesis principal, por lo cual se concluye que hay asociación moderada alta, siendo el valor $\mathrm{r}=0.563$, aproximándose a o.6, calculado con la prueba Rho de Spearman.

Segunda conclusión: En el caso de la primera hipótesis especifica $\mathrm{H}_{1}=\mathrm{La}$ inclusión educativa se relaciona significativamente con la accesibilidad en Chincha Alta 2019; fue contrastada cuando $\boldsymbol{p}$ - valor $<\mathbf{0 , 0 1}$, negándose la hipótesis nula, por lo que hay una correlación significativa entre la inclusión educativa y la accesibilidad, teniendo como resultado un $r=0.524$, resuelto por la prueba Rho de Spearman.

Tercera conclusión: La segunda hipótesis secundaria, fue obtenida también con un valor $p$ $<$ 0,01 prescindiendo de la suposición nula y aceptándose el supuesto específico, para el caso $\mathrm{H} 2$ : La inclusión educativa se relaciona significativamente con la integración laboral en Chincha Alta en el 2019, por lo que están significativamente asociadas las dimensiones inclusión educativa con integración laboral con un rango de $r=0.555$ ubicado por el estadístico Rho de Spearman.

Cuarta conclusión: En el caso de la tercera hipótesis especifica que refiere H3: La inclusión educativa se relaciona significativamente con la habilitación laboral de estudiantes con discapacidad auditiva en Chincha Alta en el 2019, aplicando los mismos parámetros aplicados al cálculo de la correlación de las hipótesis principal, primera y segunda especificas se obtuvo un coeficiente Rho de Spearman de 0,554, comprobándose la relación alta y moderada entre la inclusión educativa y la habilitación laboral del discapacitado auditivo.

El estudio tiene varias limitaciones. La más importante es que, a pesar del riguroso proceso de selección de los ítems por cada dimensión de las variables en los cuestionarios de inclusión educativa e inserción laboral respectivamente, los resultados responden a un corte en el tiempo, lo cual no permiten observar la tendencia (progresión o regresión) de las variables estudiadas, por ejemplo, desde el 2000 hasta el presente. No obstante, los hallazgos sobre la correlación positiva entre inclusión educativa e inserción laboral respecto a los post adolescentes con discapacidad auditiva pueden ayudar a impulsar cambios en su desarrollo profesional; podemos considerar que la investigación es relevante y pertinente porque nos propone un par de instrumentos para evaluar los niveles de inserción laboral e inclusión educativa, motivo por el cual se podría reaplicar los instrumentos en otros distritos y provincias del país para observar los niveles de inclusión educativa e inserción laboral en post adolescentes que poseen una discapacidad auditiva. 
Ainscow, M. y Miles, S. (2001). Making education for all inclusive: Where next? Prospects, 38(1), 15-34. Disponible en: http://dx.doi.org/10.1007/s11125-008-9055-0

Álvarez, W. (2017). Inserción laboral de personas con discapacidad en las instituciones públicas del distrito de Sicuani en el año 2016. Tesis de Licenciatura. Perú: UAC.

Blanco, R. (2011). La atención educativa a la diversidad: las escuelas inclusivas. Madrid: OEl.

Cendreno, L. (2017). La discapacidad como factor de discriminación en el ámbito laboral Tesis Doctoral. España: UCM. Recuperado de: https://eprints.ucm.es/40893/1/T38289.pdf

Cortez, J. (2015). Análisis de las principales medidas implementadas por el Estado Peruano para promover el acceso al empleo de las personas con discapacidad. Maestría en Derecho del Trabajo y de la Seguridad Social. Perú: PUCP.

Delmastro y De Montes (2015). La incorporación de la Empresa de Producción Social como proyecto educativo para la inserción socio-laboral de las personas con discapacidad de TELBCOL. España: Universidad de Córdoba. Disponible en: https://dialnet.unirioja.es/ servlet/tesis?codigo $=65990$

Echeita, G. y Ainscow, M. (2010). La educación inclusiva como derecho. Marco de referencia y pautas de acción para el desarrollo de una revolución pendiente. Revista Tejuelo. $\mathrm{N}^{\circ} 12$. Disponible en: https://repositorio.uam.es/bitstream/handle/10486/661330/educacion_ echeita_TEJUELO_2011.pdf? sequence=1\&isAllowed $=y$

Garavito, D. (2014). La inclusión de las Personas con Discapacidad en el mercado laboral colombiano, una acción conjunta. Maestría en Discapacidad e Inclusión Social. Colombia: UNC. Recuperado de: $\mathrm{http}$ ://bdigital.unal.edu.co/46075/1/52646657.2014.pdf.

Giné, C. y Font, J. (2007). El alumnado con discapacidad intelectual y del desarrollo. En Joan Bonals y Manuel Sánchez-Cano (coord.) Manual de asesoramiento pedagógico (pp. 879-914). Barcelona: Editorial Grao.

King and Edmunds (2001). Leadership for Inclusion. Critical issues in the future of learning and teaching. Canada: Brock University. from: https://www.sensepublishers.com/ media/577-leadership-for-inclusion.pdf

INEI - Instituto Nacional de Estadística e Informática del Perú (2017). Resultados definitivos del Censo XII de población, VII de vivienda, III de comunidades indígenas - Región Ica. Ediciones INEI, Tomo I, p.687 y ss. Disponible en: https://www.inei.gob.pe/media/MenuRecursivo/ publicaciones_digitales/Est/Lib1545/

Lazo, M. (2016). Acceso laboral de las personas con discapacidad visual en el Distrito Metropolitano de Quito: indicadores y estrategias que fomenten su inclusión social. Maestría en Desarrollo del control Comunitario. Ecuador: PUCE. Recuperado: http://repositorio.puce.edu.ec/ handle/22000/1230

Ley 27337 (2000). Código de los niños y adolescentes del Perú.

Disponible en: http://www2.congreso.gob.pe/sicr/cendocbib/con2_uibd. nsf/785F189E4413AAE805257662007254DA/\$FILE/PERU_LEY_27337.pdf

Ley 28044 (2003). Ley General de Educación del Perú. Disponible en: http://www.minedu. gob.pe/comunicado/pdf/normativa-2018/ley-28044/ley-28044-ley-general-deeducacion-24-11-2017.pdf

Lidón, L. (2013). Derechos humanos, discapacidad y toma de conciencia: artículo 8 de la Convención sobre los Derechos de las Personas con Discapacidad, un camino previo por recorrer. Programa de doctorado en Derechos Humanos, Democracia y Justicia Internacional. España: UV. Recuperado de: http://roderic.uv.es/bitstream/handle/10550/32238/TESIS\%20 DOCTORAL_ L LENOR\%2OLID\%C3\%93N\%2OHERAS_DEPOSITO\%2ODEF_PAPEL. pdf? squence $=1$ \&isAllowed $=y$

Marchesi, Á. (2014). Estrategias para el cambio educativo. Pensamiento Iberoamericano, 7(2), 253268. 
Moliner, O. (2013). Educación Inclusiva. Brasil: Universidad de Jaume. Recuperado de: http:// repositori.uji.es/xmlui/bitstream/handle/10234/72966/s83.pdf?sequence $=1$

Murphy, K. (2014). Teacher Attitudes Toward Inclusion Practices. As the study of teachers' attitudes towards inclusion and inclusion in general becomes even more profound, it is discovered that there is a disconnection between research and the classroom. Master of Arts in Education Faculty of Education. Canada: Mount Saint Vincent University. Recuperado de:http:// dc.msvu.ca:8080/xmlui/bitstream/handle/10587/1372/KristaMurphyMAEdThesis2014. pdf? sequence $=1$ \&isAllowed $=y$

Nussbaum, Martha (2007). Las fronteras de la justicia. Consideraciones sobre la exclusión, trad. de Ramón Vila Vernis y Albino Santos Mosquera, Paidós, Barcelona. Citado por Cuenca (2012) Revista de Estudios Políticos. Universidad Carlos III de Madrid. Recuperado de: https:// dialnet.unirioja.es/servlet/articulo?codigo $=5712189$

Parrilla, Á. (2002). La voz de la experiencia: la colaboración como estrategia de inclusión. Aula de Innovación Educativa, 121

Pérez, O. (2011). Feminismo, Liberalismo y Comunitarismo. Una aproximación a sus relaciones, implicaciones y dilemas, Sistema, 217

Pernía, M. (2019). Reacción intermodal de las cortezas auditiva y visual de la rata en un modelo de sordera bilateral a largo plazo. Tesis de Doctor en Neurociencias. Universidad de Salamanca.

Saleem, A. (2017). Teachers' attitudes towards the inclusion of children with special educational needs in Jordanian ordinary schools. PhD thesis. UK: University of Nottingham. From: prints. nottingham.ac.uk/44620/1/Saleem\%20- Coreccted\%20PhD\%20Thesis\%20-ploaded\%20 $\% 281 \% 29 \% 20 \% 281 \% 29$.pdf

Unesco (2008). Examen de la situación actual de la educación Especial. Paris: Unesco.

Vidal, R. y Cornejo, C. (2012). Empleo con apoyo: Una estrategia de inserción laboral para jóvenes con discapacidad intelectual. Revista Convergencia Educativa, 1, 113-127. Recuperado de: http://www.descubreme.cl/wp-content/uploads/2019/o4/Empleo-con-apoyo_Unaestratega-para-jo\%CC\%81venes.pdf

Vigostky L.S. (1982). De las agendas personales de L.S. Vigostky. Revista de la Universidad Estatal de Moscú. Citado por Tatiana V. Akutina en L.S. Vigostky y L.R. Luria: La formación en neuropsicología. Recuperado de: DialnetLSVigotskyYARLuria-2011215\%20(1).pdf

Walsh, M. (2018). The Inclusion of Students with Special Needs in the General Education Classroom. Senior Theses and Capstone Projects. USA: Dominican University of California. from: https://scholar.dominican.edu/senior-theses/69/

(C) Los autores. Este artículo es publicado por la Horizonte de la Ciencia de la Unidad de Posgrado de la Facultad de Educación de la Universidad Nacional del Centro del Perú. Este es un artículo de acceso abierto, distribuido bajo los términos de la Licencia Atribución-No Comercial 4.0 Internacional.(https://creativecommons.org/licenses/by-nc/4.o/), que permite el uso no comercial y distribución en cualquier medio, siempre que la obra original sea debidamente citada. 